九州大学学術情報リポジトリ

Kyushu University Institutional Repository

\title{
A Remark on the Hankel Determinant Formula for Solutions of the Toda Equation
}

Kaj iwara, Kenj i

Graduate School of Mathematics, Kyushu University

Mazzocco, Marta

School of Mathematics, The University of Manchester

Ohta, Yasuhiro

Department of Mathematics, Kobe University

http://hdl. handle. net/2324/3401

出版情報: Journal of Physics A : Mathematical and Theoretical. 40 (42), pp.12661-12675, 200710-19. Faculty of Mathematics, Kyushu University

バージョン：

権利関係: (c) 2006 Institute of Physics and IOP Publishing Limited 


\section{MHF Preprint Series}

Kyushu University

21st Century COE Program

Development of Dynamic Mathematics with

High Functionality

\section{A remark on the Hankel determinant formula for solutions of the Toda equation}

\section{K. Kajiwara, M. Mazzocco \\ Y. Ohta}

\section{MHF 2007-3}

( Received January 16, 2007 )

Faculty of Mathematics

Kyushu University

Fukuoka, JAPAN 


\title{
A Remark on the Hankel Determinant Formula for Solutions of the Toda Equation
}

\author{
Kenji Kajiwara ${ }^{1}$, Marta Mazzocco ${ }^{2}$ and Yasuhiro Ohta ${ }^{3}$ \\ ${ }^{1}$ Graduate School of Mathematics, Kyushu University, 6-10-1 Hakozaki, \\ Fukuoka 812-8581, Japan \\ ${ }^{2}$ School of Mathematics, The University of Manchester, Sackville Street, \\ Manchester M60 1QD, United Kingdom \\ ${ }^{3}$ Department of Mathematics, Kobe University, Rokko, Kobe 657-8501, Japan
}

\begin{abstract}
We consider the Hankel determinant formula of the $\tau$ functions of the Toda equation. We present a relationship between the determinant formula and the auxiliary linear problem, which is characterized by a compact formula for the $\tau$ functions in the framework of the KP theory. Similar phenomena that have been observed for the Painlevé II and IV equations are recovered. The case of finite lattice is also discussed.
\end{abstract}




\section{Introduction}

The Toda equation[24]

$$
\frac{d^{2} y_{n}}{d t^{2}}=\mathrm{e}^{y_{n-1}-y_{n}}-\mathrm{e}^{y_{n}-y_{n+1}}
$$

where $n \in \mathbb{Z}$, is one of the most important integrable systems. It can be expressed in various forms such as

$$
\begin{array}{ll}
\frac{d V_{n}}{d t}=V_{n}\left(I_{n}-I_{n+1}\right), & \frac{d I_{n}}{d t}=V_{n-1}-V_{n}, \\
\frac{d \alpha_{n}}{d t}=\alpha_{n}\left(\beta_{n+1}-\beta_{n}\right), & \frac{d \beta_{n}}{d t}=2\left(\alpha_{n}^{2}-\alpha_{n-1}^{2}\right),
\end{array}
$$

where the dependent variables are related to $y_{n}$ as

$$
V_{n}=\mathrm{e}^{y_{n}-y_{n+1}}, \quad I_{n}=\frac{d y_{n}}{d t}, \quad \alpha_{n}=\frac{1}{2} \mathrm{e}^{\frac{y_{n}-y_{n+1}}{2}}, \quad \beta_{n}=-\frac{1}{2} \frac{d y_{n}}{d t} .
$$

The Toda equation can be reduced to the bilinear equation

$$
\tau_{n}^{\prime \prime} \tau_{n}-\left(\tau_{n}^{\prime}\right)^{2}=\tau_{n+1} \tau_{n-1},
$$

by the dependent variable transformation

$$
y_{n}=\log \frac{\tau_{n-1}}{\tau_{n}}, \quad V_{n}=\frac{\tau_{n+1} \tau_{n-1}}{\tau_{n}^{2}}, \quad I_{n}=\frac{d}{d t} \log \frac{\tau_{n-1}}{\tau_{n}} .
$$

In general, the determinant structure of the $\tau$ function (dependent variable of bilinear equation) is the characteristic property of integrable systems. For example, the Casorati determinant formula of the N-soliton solution of the Toda equation (see, for example, $[25,4])$

$\tau_{n}=e^{\frac{t^{2}}{2}} \operatorname{det}\left(f_{n+j-1}^{(i)}\right)_{i, j=1, \ldots, N}, \quad f_{n}^{(k)}=p_{k}^{n} e^{p_{k} t+\eta_{k 0}}+p_{k}^{-n} e^{\frac{1}{p_{k}} t+\xi_{k 0}}$,

where $p_{k}, \eta_{k 0}$ and $\xi_{k 0}(k=1, \ldots, N)$ are constants, is a direct consequence of the Sato theory; the solution space of soliton equations is the universal Grassman manifold, on which infinite dimensional Lie algebras are acting $[9,15,25]$.

If we consider the Toda equation on semi-infinite or finite lattice, the soliton solutions do not exist but another determinantal solution arises. For the semi-infinite case we impose the boundary condition as

$$
\tau_{-1}=0, \quad \tau_{0}=1, \quad V_{0}=0, \quad n \geq 0 .
$$

Then $\tau_{n}$ admits the Hankel determinant formula[13, 5, 6]

$$
\tau_{n}=\operatorname{det}\left(a_{i+j-2}\right)_{i, j=1, \ldots, n}, \quad a_{0}=\tau_{1}, \quad a_{i}=a_{i-1}^{\prime}, \quad n \in \mathbb{Z}_{\geq 0} .
$$

The important feature of this determinant formula is that the lattice site $n$ appears as the determinant size, while for the soliton solutions the determinant size describes the number of solitons. This type of determinant formula is actually a special case of the determinant forula for the infinite lattice[12]. However the meaning of the formula has not been yet fully understood.

The purpose of this article is to establish a characterization of the Hankel determinant formula of the Toda equation; entries of the matrices in the determinant formula are closely related to the solution of auxiliary linear problem. Moreover, this relationship can be described by a compact formula in the framework of the theory of KP hierarchy.

In section 2, we discuss the Hankel determinant formula of the infinite Toda equation and present the relationship between the determinant formula and auxiliary linear problem. In section 3, we apply the results to the Painlevé II equation. We consider the case of finite lattice in section 4 . 


\section{Hankel determinant formula of the solution of the Toda equation}

\subsection{Determinant formula and auxiliary linear problem}

The Hankel determinant formula for $\tau_{n}$ satisfying the infinite Toda equation (1.5) is given by as follows:

Proposition 2.1 [12] For fixed $k \in \mathbb{Z}$, we have:

$$
\begin{aligned}
& \frac{\tau_{k+n}}{\tau_{k}}= \begin{cases}\operatorname{det}\left(a_{i+j-2}^{(k)}\right)_{i, j=1, \ldots, n} & n>0, \\
1 & n=0, \\
\operatorname{det}\left(b_{i+j-2}^{(k)}\right)_{i, j=1, \ldots,|n|} & n<0,\end{cases} \\
& \left\{\begin{array}{l}
a_{i}^{(k)}=a_{i-1}^{(k) \prime}+\frac{\tau_{k-1}}{\tau_{k}} \sum_{l=0}^{i-2} a_{l}^{(k)} a_{i-2-l}^{(k)}, \quad a_{0}^{(k)}=\frac{\tau_{k+1}}{\tau_{k}}, \\
b_{i}^{(k)}=b_{i-1}^{(k) \prime}+\frac{\tau_{k+1}}{\tau_{k}} \sum_{l=0}^{i-2} b_{l}^{(k)} b_{i-2-l}^{(k)}, \quad b_{0}^{(k)}=\frac{\tau_{k-1}}{\tau_{k}} .
\end{array}\right.
\end{aligned}
$$

We shall now relate the determinant formula to the auxiliary linear problem of the Toda equation (1.2) given by

$$
\left\{\begin{array}{l}
V_{n-1} \Psi_{n-1}+I_{n} \Psi_{n}+\Psi_{n+1}=\lambda \Psi_{n}, \\
\frac{d \Psi_{n}}{d t}=V_{n-1} \Psi_{n-1}
\end{array}\right.
$$

or

$$
\left\{\begin{array}{l}
L_{n} \Psi_{n}=\lambda \Psi_{n} \\
\frac{d \Psi_{n}}{d t}=B_{n} \Psi_{n}
\end{array}\right.
$$

where

$$
L_{n}=V_{n-1} e^{-\partial_{n}}+I_{n}+e^{\partial_{n}}, \quad B_{n}=V_{n-1} e^{-\partial_{n}} .
$$

The adjoint linear problem associated with the linear problem (2.3) is given by

$$
\left\{\begin{array}{l}
\Psi_{n-1}^{*}+I_{n} \Psi_{n}^{*}+V_{n} \Psi_{n+1}^{*}=\lambda \Psi_{n}^{*} \\
\frac{d \Psi_{n}^{*}}{d t}=-V_{n} \Psi_{n+1}^{*}
\end{array}\right.
$$

or

$$
\left\{\begin{array}{l}
L_{n}^{*} \Psi_{n}^{*}=\lambda \Psi_{n}^{*}, \\
-\frac{d \Psi_{n}^{*}}{d t}=B_{n}^{*} \Psi_{n}^{*}
\end{array}\right.
$$

where

$$
L_{n}^{*}=V_{n} e^{\partial_{n}}+I_{n}+e^{-\partial_{n}}, \quad B_{n}^{*}=V_{n} e^{\partial_{n}} .
$$

The compatibility condition for each problem

$$
\frac{d L_{n}}{d t}=\left[B_{n}, L_{n}\right], \quad \frac{d L_{n}^{*}}{d t}=\left[-B_{n}^{*}, L_{n}^{*}\right]
$$

yields the Toda equation (1.2), respectively.

One of our main results is that the entries of the determinants in the Hankel determinant formula arise as the coefficients of asymptoric expansions at $\lambda=\infty$ of 
the ratio of solutions of the linear and adjoint linear problems. To state the result more precisely, we define

$$
\Xi_{k}(t, \lambda)=\frac{\Psi_{k}(t, \lambda)}{\Psi_{k+1}(t, \lambda)}, \quad \Omega_{k}(t, \lambda)=\frac{\Psi_{k+1}^{*}(t, \lambda)}{\Psi_{k}^{*}(t, \lambda)} .
$$

Theorem 2.2 (i) The ratios $\Xi_{k}(t, \lambda)$ and $\Omega_{k}(t, \lambda)$ admits two kinds of asymptotic expansions as functions of $\lambda$ as $\lambda \rightarrow \infty$

$$
\begin{aligned}
& \Xi_{k}^{(-1)}(t, \lambda)=u_{-1} \lambda^{-1}+u_{-2} \lambda^{-2}+\cdots, \\
& \Xi_{k}^{(1)}(t, \lambda)=v_{1} \lambda+v_{0}+v_{-1} \lambda^{-1}+\cdots,
\end{aligned}
$$

and

$$
\begin{aligned}
& \Omega_{k}^{(-1)}(t, \lambda)=u_{-1} \lambda^{-1}+u_{-2} \lambda^{-2}+\cdots, \\
& \Omega_{k}^{(1)}(t, \lambda)=v_{1} \lambda+v_{0}+v_{-1} \lambda^{-1}+\cdots,
\end{aligned}
$$

respectively.

(ii) The above asymptotic expantions are related to the Hankel determinants entries $a_{i}^{(k)}$ and $b_{i}^{(k)}$ as follows:

$\Xi_{k}^{(-1)}(t, \lambda)=\frac{1}{\lambda} \frac{\tau_{k}}{\tau_{k-1}} \sum_{i=0}^{\infty} b_{i}^{(k)} \lambda^{-i}$,

$\Xi_{k}^{(1)}(t, \lambda)=\frac{\tau_{k}^{2}}{\tau_{k+1} \tau_{k-1}}\left[\lambda-\frac{\left(\frac{\tau_{k}}{\tau_{k+1}}\right)^{\prime}}{\frac{\tau_{k}}{\tau_{k+1}}}-\frac{1}{\lambda} \frac{\tau_{k}}{\tau_{k+1}} \sum_{i=0}^{\infty} a_{i}^{(k+1)}(-\lambda)^{-i}\right]$,

and

$$
\begin{aligned}
& \Omega_{k}^{(-1)}(t, \lambda)=\frac{1}{\lambda} \frac{\tau_{k}}{\tau_{k+1}} \sum_{i=0}^{\infty} a_{i}^{(k)}(-\lambda)^{-i} \\
& \Omega_{k}^{(1)}(t, \lambda)=\frac{\tau_{k}^{2}}{\tau_{k+1} \tau_{k-1}}\left[\lambda-\frac{\left(\frac{\tau_{k-1}}{\tau_{k}}\right)^{\prime}}{\frac{\tau_{k-1}}{\tau_{k}}}-\frac{1}{\lambda} \frac{\tau_{k}}{\tau_{k-1}} \sum_{i=0}^{\infty} b_{i}^{(k-1)} \lambda^{-i}\right]
\end{aligned}
$$

(iii) $\Xi_{k}^{( \pm 1)}$ and $\Omega_{k}^{( \pm 1)}$ are related as follows:

$$
\Omega_{k}^{(1)}(t, \lambda) \Xi_{k}^{(-1)}(t, \lambda)=\frac{\tau_{k}^{2}}{\tau_{k+1} \tau_{k-1}}, \quad \Omega_{k}^{(-1)}(t, \lambda) \Xi_{k}^{(1)}(t, \lambda)=\frac{\tau_{k}^{2}}{\tau_{k+1} \tau_{k-1}} .
$$

Brief sketch of the proof of Theorem 2.2. One can prove Theorem 2.2 by direct calculation. From the linear problem (2.3) and (2.10), we see that $\Xi_{k}(t, \lambda)$ satisfies the Riccati equation

$$
\frac{\partial \Xi_{k}}{\partial t}=-V_{k} \Xi_{k}^{2}+\left(\lambda-I_{k}\right) \Xi_{k}-1
$$

Plugging series expansion $\Xi_{k}=\lambda^{\rho} \sum_{i=0}^{\infty} h_{i} \lambda^{-i}$ into (2.20) and considering the balance of leading terms, we find that $\rho$ must be $\rho=1,-1$, which proves (2.11) and (2.12) . Moreover, it is possible to verify (2.15) and (2.16) by deriving recursion relations of coefficients for each case and comparing them with (2.2). Similarly, from the Riccati equation for $\Omega$

$$
\frac{\partial \Omega_{k}}{\partial t}=V_{k} \Omega_{k}^{2}+\left(-\lambda+I_{k+1}\right) \Omega_{k}+1
$$


one can prove the statements for $\Omega$. For (iii), putting $X_{k}=\frac{\tau_{k}^{2}}{\tau_{k+1} \tau_{k-1}} \frac{1}{\Xi_{k}^{(-1)}}=\frac{1}{V_{k} \Xi_{k}^{(-1)}}$, plugging this expression into the Riccati equation (2.21) and using (1.2), we find that $X_{k}$ satisfies (2.20). Since the expansion of $\Xi_{k}^{(-1)}$ starts from $\lambda^{-1}$, the leading order of $X_{k}$ is $\lambda$ and thus $X_{k}=\Omega^{(1)}$. The second equation of (2.19) can be proved in a similar manner.

\subsection{KP theory}

The results in the previous section can be characterized by a compact formula in terms of the language of the KP theory [9, 15, 19].

We introduce infinitely many independent variables $x=\left(x_{1}, x_{2}, x_{3}, \ldots\right), x_{1}=t$, and let $\tau_{n}(x)$ be the $\tau$ function of the one-dimensional Toda lattice hierarchy[25,9] and the first modified KP hierarchy[9]. Namely, $\tau_{n}, n \mathbb{Z}$, satisfy the following bilinear equations

$$
\begin{aligned}
& D_{x_{1}} p_{j+1}\left(\frac{1}{2} \tilde{D}\right) \tau_{n} \cdot \tau_{n}=p_{j}\left(\frac{1}{2} \tilde{D}\right) \tau_{n+1} \cdot \tau_{n-1}, \quad j=0,1,2 \ldots \\
& {\left[D_{x_{1}} p_{j}\left(\frac{1}{2} \tilde{D}\right)-p_{j+1}\left(\frac{1}{2} \tilde{D}\right)+p_{j+1}\left(-\frac{1}{2} \tilde{D}\right)\right] \tau_{n+1} \cdot \tau_{n}=0, \quad j=0,1,2 \ldots}
\end{aligned}
$$

where $p_{0}(x), p_{1}(x), \cdots$ are the elementary Schur functions

$$
\sum_{n=0}^{\infty} p_{n}(x) \kappa^{n}=\exp \sum_{i=1}^{\infty} x_{i} \kappa^{i}
$$

and

$$
\tilde{D}=\left(D_{x_{1}}, \frac{1}{2} D_{x_{2}}, \ldots, \frac{1}{n} D_{x_{n}}, \ldots\right),
$$

$D_{x_{i}}(i=1,2, \ldots)$ being the Hirota's $D$-operator. Then we have the following formula:

Proposition 2.3 For fixed $k \in \mathbb{Z}$, we have

$$
\frac{\tau_{k+n}}{\tau_{k}}= \begin{cases}\operatorname{det}\left(a_{i+j-2}^{(k)}\right)_{i, j=1, \ldots, n} & n>0 \\ 1 & n=0 \\ \operatorname{det}\left(b_{i+j-2}^{(k)}\right)_{i, j=1, \ldots,|n|} & n<0\end{cases}
$$

where

$$
a_{i}^{(k)}=p_{i}(\tilde{\partial}) \frac{\tau_{k+1}}{\tau_{k}}, \quad b_{i}^{(k)}=(-1)^{i} p_{i}(-\tilde{\partial}) \frac{\tau_{k-1}}{\tau_{k}},
$$

and

$$
\tilde{\partial}=\left(\partial_{x_{1}}, \frac{1}{2} \partial_{x_{2}}, \ldots, \frac{1}{n} \partial_{x_{n}}, \ldots\right) .
$$

Remark 2.4 It might be interesting to remark here that $a_{0}^{(k)}=\frac{\tau_{k+1}}{\tau_{k}}$ and $b_{0}^{(k)}=\frac{\tau_{k-1}}{\tau_{k}}$ satisfy the nonlinear Schrödinger hierarchy. In fact, Equations (2.2) and (2.27) with $i=2$ imply for $a=a_{0}^{(k)}$ and $b=b_{0}^{(k)}$

$$
a_{x_{2}}=a_{x_{1} x_{1}}+2 a^{2} b, \quad b_{x_{2}}=-\left(b_{x_{1} x_{1}}+2 a^{2} b\right) .
$$

Similarly, for $i=3$ we have

$$
a_{x_{3}}=a_{x_{1} x_{1} x_{1}}+6 a b a_{x_{1}}, \quad b_{x_{3}}=b_{x_{1} x_{1} x_{1}}+6 a b b_{x_{1}} .
$$


Before proceeding to the proof, we note that the auxiliary linear problem (2.3) and its adjoint problem (2.6) are recovered from the bilinear equations (2.22) and (2.23). In fact, suppose that $\tau_{n}$ depends on a discrete independent variable $l$ and satisfies the discrete modified KP equation

$$
\begin{aligned}
& D_{x_{1}} \tau_{n}(l+1) \cdot \tau_{n}(l)=-\frac{1}{\lambda} \tau_{n+1}(l+1) \tau_{n-1}(l), \\
& \left(\frac{1}{\lambda} D_{x_{1}}+1\right) \tau_{n+1}(l+1) \cdot \tau_{n}(l)-\tau_{n}(l+1) \tau_{n+1}(l)=0,
\end{aligned}
$$

then one can show that Equations (2.22) and (2.23) are equivalent to (2.31) and (2.32), respectively, through the Miwa transformation[14, 9]

$x_{n}=\frac{l}{n(-\lambda)^{n}} \quad$ or $\quad \frac{\partial}{\partial l}=-\frac{1}{\lambda} \frac{\partial}{\partial x_{1}}+\frac{1}{2 \lambda^{2}} \frac{\partial}{\partial x_{2}}+\cdots+\frac{1}{j(-\lambda)^{j}} \frac{\partial}{\partial x_{j}}+\cdots$.

Putting

$$
\begin{aligned}
& \Psi_{n}^{*}=\lambda^{-n} \frac{\tau_{n}(l+1)}{\tau_{n}(l)}, \\
& V_{n}=\frac{\tau_{n+1}(l) \tau_{n-1}(l)}{\tau_{n}(l)^{2}}, \quad I_{n}=\frac{d}{d t} \log \frac{\tau_{n-1}(l)}{\tau_{n}(l)},
\end{aligned}
$$

and noticing $t=x_{1}$, the bilinear equations (2.31) and (2.32) are rewritten as

$$
\begin{aligned}
& \Psi_{n}^{* \prime}=-V_{n+1} \Psi_{n+1}^{*}, \\
& \Psi_{n}^{*}+I_{n+1} \Psi_{n+1}^{*}+V_{n+2} \Psi_{n+2}^{*}=\lambda \Psi_{n+1}^{*},
\end{aligned}
$$

which are equivalent to the adjoint linear problem (2.6). Similarly, shifting $l \rightarrow l-1$ in (2.31) and (2.32) and putting

$$
\Psi_{n+1}=\lambda^{n} \frac{\tau_{n}(l-1)}{\tau_{n}(l)},
$$

we obtain

$$
\begin{aligned}
& \Psi_{n+1}^{\prime}=V_{n} \Psi_{n}, \\
& V_{n} \Psi_{n}+I_{n+1} \Psi_{n+1}+\Psi_{n+2}=\lambda \Psi_{n+1},
\end{aligned}
$$

which is also equivalent to the linear problem (2.3).

Proof of Proposition 2.3. From (2.37), (2.33) and (2.24) we have

$$
\begin{aligned}
& \frac{\Psi_{k}(t, \lambda)}{\Psi_{k+1}(t, \lambda)}=\frac{1}{\lambda} \frac{\tau_{k-1}(l-1) \tau_{k}(l)}{\tau_{k}(l-1) \tau_{k-1}(l)}=\frac{1}{\lambda} \frac{\tau_{k}(l)}{\tau_{k-1}(l)} \mathrm{e}^{-\frac{\partial}{\partial l}} \frac{\tau_{k-1}(l)}{\tau_{k}(l)} \\
= & \frac{1}{\lambda} \frac{\tau_{k}(l)}{\tau_{k-1}(l)} \mathrm{e}^{-\sum_{j=1}^{\infty} \frac{1}{j(-\lambda)^{j}} \frac{\partial}{\partial x_{j}}} \frac{\tau_{k-1}(l)}{\tau_{k}(l)}=\frac{1}{\lambda} \frac{\tau_{k}(l)}{\tau_{k-1}(l)} \sum_{n=0}^{\infty} p_{n}(-\tilde{\partial}) \frac{\tau_{k-1}(l)}{\tau_{k}(l)}(-\lambda)^{-n} .
\end{aligned}
$$

Therefore Equation (2.15) in Theorem 2.2 implies

$$
b_{n}^{(k)}=(-1)^{n} p_{n}(-\tilde{\partial}) \frac{\tau_{k}(l)}{\tau_{k-1}(l)} .
$$

Similarly we have from $(2.34),(2.33)$ and $(2.24)$

$$
\begin{aligned}
& \frac{\Psi_{k+1}^{*}(t, \lambda)}{\Psi_{k}^{*}(t, \lambda)}=\frac{1}{\lambda} \frac{\tau_{k+1}(l+1)}{\tau_{k}(l+1)} \frac{\tau_{k}(l)}{\tau_{k+1}(l)}=\frac{1}{\lambda} \frac{\tau_{k}(l)}{\tau_{k+1}(l)} \mathrm{e}^{\frac{\partial}{\partial l}} \frac{\tau_{k+1}(l)}{\tau_{k}(l)} \\
= & \frac{1}{\lambda} \frac{\tau_{k}(l)}{\tau_{k+1}(l)} \mathrm{e}^{\sum_{j=1}^{\infty} \frac{1}{j(-\lambda)^{j}} \frac{\partial}{\partial x_{j}}} \frac{\tau_{k+1}(l)}{\tau_{k}(l)}=\frac{1}{\lambda} \frac{\tau_{k}(l)}{\tau_{k+1}(l)} \sum_{n=0}^{\infty} p_{n}(\tilde{\partial}) \frac{\tau_{k+1}(l)}{\tau_{k}(l)}(-\lambda)^{-n} .
\end{aligned}
$$


Therefore comparing with (2.17) we obtain

$$
a_{n}^{(k)}=p_{n}(\tilde{\partial}) \frac{\tau_{k+1}(l)}{\tau_{k}(l)},
$$

which proves Proposition 2.3.

\section{Painlevé equations}

\subsection{Local Lax pair}

Originally the relations between determinant formula of the solutions and auxiliary linear problem have been derived for the Painlevé II and IV equations [10, 11]. In the particular case of the rational solutions of the Painlevé II and IV equations these results give the relation between determinant formula and the Airy function found in $[7,3]$. It may be natural to regard those relationships as originating from the Toda equation, since the sequence of $\tau$ functions generated by the Bäcklund transformations of Painlevé equations are described by the Toda equation $[20,21,22,23,8,12]$. In this section, we show that the results for the Painlevé II equation can be recovered from the results in the section 2. The key ingredient of the correspondence is the local Lax pair, which is the auxiliary linear problem for Toda equation formulated by a pair of $2 \times 2$ matrices[1]:

$$
\begin{gathered}
\widetilde{L}_{n} \phi_{n}=\phi_{n+1}, \quad \widetilde{L}_{n}(t, \lambda)=\left(\begin{array}{cc}
-I_{n}+\lambda & -\mathrm{e}^{-y_{n}} \\
\mathrm{e}^{y_{n}} & 0
\end{array}\right) \\
\frac{d \phi_{n}}{d t}=\widetilde{B}_{n} \phi_{n}, \quad \widetilde{B}_{n}(t, \lambda)=\left(\begin{array}{cc}
-\frac{1}{2} & 0 \\
0 & \frac{1}{2}
\end{array}\right) \lambda+\left(\begin{array}{cc}
0 & \mathrm{e}^{-y_{n}} \\
-\mathrm{e}^{y_{n-1}} & 0
\end{array}\right) \\
\phi_{n}=\left(\begin{array}{c}
\phi_{n}^{(1)} \\
\phi_{n}^{(2)}
\end{array}\right), \quad y_{n}=\log \frac{\tau_{n-1}}{\tau_{n}} .
\end{gathered}
$$

Similarly, the adjoint linear problem is given by

$$
\begin{array}{cl}
\widetilde{L}_{n}^{*} \phi_{n}^{*}=\phi_{n-1}^{*}, & \widetilde{L}_{n}^{*}(t, \lambda)=\left(\begin{array}{cc}
-I_{n}+\lambda & \mathrm{e}^{y_{n}} \\
-\mathrm{e}^{-y_{n}} & 0
\end{array}\right) \\
\frac{d \phi_{n}^{*}}{d t}=\widetilde{B}_{n}^{*} \phi_{n}^{*}, & \widetilde{B}_{n}^{*}(t, \lambda)=\left(\begin{array}{cc}
\frac{1}{2} & 0 \\
0 & -\frac{1}{2}
\end{array}\right) \lambda+\left(\begin{array}{cc}
0 & \mathrm{e}^{y_{n}} \\
-\mathrm{e}^{-y_{n+1}} & 0
\end{array}\right) \\
& \phi_{n}^{*}=\left(\begin{array}{c}
\phi_{n}^{*(1)} \\
\phi_{n}^{*(2)}
\end{array}\right) .
\end{array}
$$

Compatibility condition for each problem

$$
\frac{d \widetilde{L}_{n}}{d t}=\widetilde{B}_{n+1} \widetilde{L}_{n}-\widetilde{L}_{n} \widetilde{B}_{n}, \quad \frac{d \widetilde{L}_{n}^{*}}{d t}=-\widetilde{B}_{n-1}^{*} \widetilde{L}_{n}^{*}+\widetilde{L}_{n}^{*} \widetilde{B}_{n}^{*}
$$

gives Toda equation (1.2), respectively. By comparing (3.1) and (3.2) with (2.3), similarly by comparing (3.4) and (3.5) with (2.6), one sees that there is a relationship between the solutions of the linear problems:

$$
\begin{array}{ll}
\phi_{n}^{(1)}=\mathrm{e}^{-\frac{1}{2} \lambda t} \Psi_{n}, & \phi_{n}^{(2)}=\mathrm{e}^{-\frac{1}{2} \lambda t} \frac{\tau_{n-2}}{\tau_{n-1}} \Psi_{n-1}, \\
\phi_{n}^{*(1)}=\mathrm{e}^{\frac{1}{2} \lambda t} \Psi_{n}^{*}, & \phi_{n}^{*(2)}=-\mathrm{e}^{\frac{1}{2} \lambda t} \frac{\tau_{n+1}}{\tau_{n}} \Psi_{n+1}^{*} .
\end{array}
$$




\subsection{Painlevé II equation}

In this section we consider the Painlevé II equation $\left(\mathrm{P}_{\mathrm{II}}\right)$

$$
\frac{d^{2} u}{d t^{2}}=2 u^{3}-4 t u+4\left(\alpha+\frac{1}{2}\right)
$$

We denote (3.10) as $\mathrm{P}_{\mathrm{II}}[\alpha]$ when it is necessary to specify the parameter $\alpha$ explicitly. Suppose that $\tau_{0}$ and $\tau_{1}$ satisfy the bilinear equations

$$
\begin{aligned}
& \left(D_{t}^{2}-2 t\right) \tau_{1} \cdot \tau_{0}=0, \\
& \left(D_{t}^{3}-2 t D_{t}-4\left(\alpha+\frac{1}{2}\right)\right) \tau_{1} \cdot \tau_{0}=0,
\end{aligned}
$$

then it is easily verified that

$$
u=\frac{d}{d t} \log \frac{\tau_{1}}{\tau_{0}}
$$

satisfies $\mathrm{P}_{\mathrm{II}}[\alpha](3.10)$. If we generate the sequence $\tau_{n}(n \in \mathbb{Z})$ by the Toda equation

$$
\frac{1}{2} D_{t}^{2} \tau_{n} \cdot \tau_{n}=\tau_{n+1} \tau_{n-1}
$$

then it is shown that $\tau_{n}$ satisfy

$$
\begin{aligned}
& \left(D_{t}^{2}-2 t\right) \tau_{n+1} \cdot \tau_{n}=0 \\
& \left(D_{t}^{3}-2 t D_{t}-4\left(\alpha+\frac{1}{2}+n\right)\right) \tau_{n+1} \cdot \tau_{n}=0
\end{aligned}
$$

and that

$$
u=\frac{d}{d t} \log \frac{\tau_{n+1}}{\tau_{n}}
$$

satisfies $\mathrm{P}_{\mathrm{II}}[\alpha+n]$. In this sense, the Toda equation (3.14) describes the Bäcklund transformation of $\mathrm{P}_{\mathrm{II}}$ (see, for example, [18]). Therefore one can apply Proposition 2.1 to obtain the determinant formula: for fixed $k \in \mathbb{Z}$ we have

where

$$
\frac{\tau_{k+n}}{\tau_{k}}= \begin{cases}\operatorname{det}\left(a_{i+j-2}^{(k)}\right)_{i, j=1, \ldots, n} & n>0 \\ 1 & n=0 \\ \operatorname{det}\left(b_{i+j-2}^{(k)}\right)_{i, j=1, \ldots,|n|} & n<0\end{cases}
$$

$$
\begin{cases}a_{i}^{(k)}=a_{i-1}^{(k) \prime}+\frac{\tau_{k-1}}{\tau_{k}} \sum_{l=0}^{i-2} a_{l}^{(k)} a_{i-2-l}^{(k)}, & a_{0}^{(k)}=\frac{\tau_{k+1}}{\tau_{k}}, \\ b_{i}^{(k)}=b_{i-1}^{(k) \prime}+\frac{\tau_{k+1}}{\tau_{k}} \sum_{l=0}^{i-2} b_{l}^{(k)} b_{i-2-l}^{(k)}, & b_{0}^{(k)}=\frac{\tau_{k-1}}{\tau_{k}} .\end{cases}
$$

Now consider the auxiliary linear problem for $\mathrm{P}_{\mathrm{II}}[\alpha](3.10)[8]$ :

$$
\begin{aligned}
\frac{\partial Y}{\partial \lambda}=A Y, \quad A=\left(\begin{array}{cc}
\frac{1}{4} & 0 \\
0 & -\frac{1}{4}
\end{array}\right) \lambda^{2} & +\left(\begin{array}{cc}
0 & -\frac{1}{2} \frac{\tau_{1}}{\tau_{0}} \\
\frac{1}{2} \frac{\tau_{-1}}{\tau_{0}} & 0
\end{array}\right) \lambda \\
& +\left(\begin{array}{cc}
-\frac{z+t}{2} & \frac{1}{2}\left(\frac{\tau_{1}}{\tau_{0}}\right)^{\prime} \\
\frac{1}{2}\left(\frac{\tau_{-1}}{\tau_{0}}\right)^{\prime} & \frac{z+t}{2}
\end{array}\right), \\
0 & \frac{\partial Y}{\partial t}=B Y, \quad B=\left(\begin{array}{cc}
-\frac{1}{2} & 0 \\
0 & \frac{1}{2}
\end{array}\right) \lambda+\left(\begin{array}{cc}
\tau_{-1} \\
-\frac{\tau_{0}}{\tau_{0}} & 0
\end{array}\right)
\end{aligned}
$$




$$
Y=\left(\begin{array}{c}
Y_{1} \\
Y_{2}
\end{array}\right), \quad z=-\frac{\tau_{1} \tau_{-1}}{\tau_{0}^{2}} .
$$

Comparing (3.21) with (3.2), we immediately find that

$$
B=\widetilde{B_{1}}, \quad Y=\phi_{1} .
$$

We note that it is possible to regard (3.20) as the equation defining $\lambda$-flow which is consistent with evolution in $t$. Also, the linear equation (3.1) describes the Bäcklund transformation. Similarly, we have the adjoint problem

$$
\begin{aligned}
& \frac{\partial Y^{*}}{\partial \lambda}=A^{*} Y^{*}, A^{*}=\left(\begin{array}{cc}
\frac{1}{4} & 0 \\
0 & -\frac{1}{4}
\end{array}\right) \lambda^{2}+\left(\begin{array}{cc}
0 & \frac{1}{2} \frac{\tau_{-1}}{\tau_{0}} \\
-\frac{1}{2} \frac{\tau_{1}}{\tau_{0}} & 0
\end{array}\right) \lambda \\
& +\left(\begin{array}{cc}
-\frac{z+t}{2} & \frac{1}{2}\left(\frac{\tau_{-1}}{\tau_{0}}\right)^{\prime} \\
\frac{1}{2}\left(\frac{\tau_{1}}{\tau_{0}}\right)^{\prime} & \frac{z+t}{2}
\end{array}\right) \\
& \frac{\partial Y^{*}}{\partial t}=B^{*} Y^{*}, \quad B^{*}=\left(\begin{array}{cc}
\frac{1}{2} & 0 \\
0 & -\frac{1}{2}
\end{array}\right) \lambda+\left(\begin{array}{cc}
0 & \frac{\tau_{-1}}{\tau_{0}} \\
-\frac{\tau_{1}}{\tau_{0}} & 0
\end{array}\right), \\
& Y^{*}=\left(\begin{array}{c}
Y_{1} \\
Y_{2}
\end{array}\right), \quad z=-\frac{\tau_{1} \tau_{-1}}{\tau_{0}^{2}},
\end{aligned}
$$

where we have a correspondence

$$
B^{*}=\widetilde{B}_{0}^{*}, \quad Y^{*}=\phi_{1}^{*}
$$

Therefore, if we apply Theorem 2.2 noticing (3.8) and (3.9), we have the following:

Proposition 3.1 We put

$$
\Lambda(t, \lambda)=\frac{Y_{2}}{Y_{1}}, \quad \Pi(t, \lambda)=\frac{Y_{2}^{*}}{Y_{1}^{*}} .
$$

(i) The ratios $\Lambda$ and $\Pi$ admit two kinds of asymptotic expansions as functions of $\lambda$ as $\lambda \rightarrow \infty$

$$
\begin{aligned}
& \Lambda^{(-1)}(t, \lambda)=u_{-1} \lambda^{-1}+u_{-2} \lambda^{-2}+\cdots \\
& \Lambda^{(1)}(t, \lambda)=v_{1} \lambda+v_{0}+v_{-1} \lambda^{-1}+\cdots
\end{aligned}
$$

and

$$
\begin{aligned}
& \Pi^{(-1)}(t, \lambda)=u_{-1} \lambda^{-1}+u_{-2} \lambda^{-2}+\cdots \\
& \Pi^{(1)}(t, \lambda)=v_{1} \lambda+v_{0}+v_{-1} \lambda^{-1}+\cdots
\end{aligned}
$$

respectively.

(ii) The above asymptotic expansions are related to the Hankel determinants entries $a_{i}^{(k)}$ and $b_{i}^{(k)}$ as follows:

$$
\begin{aligned}
& \Lambda^{(-1)}(t, \lambda)=\frac{1}{\lambda} \sum_{i=0}^{\infty} b_{i}^{(0)} \lambda^{-i}, \\
& \Lambda^{(1)}(t, \lambda)=\frac{\tau_{0}}{\tau_{1}}\left[\lambda-\frac{\left(\frac{\tau_{0}}{\tau_{1}}\right)^{\prime}}{\frac{\tau_{0}}{\tau_{1}}}-\frac{1}{\lambda} \frac{\tau_{0}}{\tau_{1}} \sum_{i=0}^{\infty} a_{i}^{(1)}(-\lambda)^{-i}\right],
\end{aligned}
$$




$$
\begin{aligned}
& \text { and } \\
& \Pi^{(-1)}(t, \lambda)=\frac{1}{(-\lambda)} \sum_{i=0}^{\infty} a_{i}^{(0)}(-\lambda)^{-i}, \\
& \Pi^{(1)}(t, \lambda)=-\frac{\tau_{0}}{\tau_{-1}}\left[\lambda-\frac{\left(\frac{\tau_{-1}}{\tau_{0}}\right)^{\prime}}{\frac{\tau_{-1}}{\tau_{0}}}-\frac{1}{\lambda} \frac{\tau_{0}}{\tau_{-1}} \sum_{i=0}^{\infty} b_{i}^{(-1)} \lambda^{-i}\right] .
\end{aligned}
$$

(iii) $\Lambda^{( \pm 1)}$ and $\Pi^{( \pm 1)}$ are related as follows:

$$
\Pi^{(1)}(t, \lambda) \Lambda^{(-1)}(t, \lambda)=1, \quad \Pi^{(-1)}(t, \lambda) \Lambda^{(1)}(t, \lambda)=1 .
$$

Proposition 3.1 is equivalent to the results presented in $[7,10]$. In other words, the relations between determinant formula for the solution of $\mathrm{P}_{\mathrm{II}}$ and auxiliary linear problems originate from the structure of the Toda equation. We also note that one can recover the results for the Painlevé IV equation[3, 11] in similar manner.

\section{Toda equation on finite lattice}

\subsection{Determinant formula}

Let us consider the Toda equation on the finite lattice. Namely, we impose the boundary condition

$$
\begin{array}{ll}
V_{0}=0, & V_{N}=0, \\
y_{0}=-\infty, & y_{N+1}=\infty, \\
\alpha_{0}=0, & \alpha_{N}=0,
\end{array}
$$

on the Toda equation (1.1), (1.2) and (1.3), respectively. In order to realize this condition on the level of the $\tau$ funtion, we proceed as follows: in the bilinear equation (1.5), imposing the boundary condition on the left edge of lattice

$$
\tau_{-1}=0, \quad \tau_{0} \neq 0,
$$

it immediately follows $\tau_{-2}=0$ and one can restrict the Toda equation on the semiinfinite lattice $n \geq 0$. In this case, the determinant formula reduces to

$$
\frac{\tau_{k}}{\tau_{0}}=\operatorname{det}\left(a_{i+j-2}^{(0)}\right)_{i, j=1, \cdots, k}(n \geq 1), \quad a_{i+1}^{(0)}=a_{i}^{(0) \prime}, \quad a_{0}^{(0)}=\frac{\tau_{1}}{\tau_{0}},
$$

which is equivalent to (1.9). Moreover, imposing the boundary condition on the right edge of lattice

$$
\tau_{N} \neq 0, \quad \tau_{N+1}=0,
$$

then we have the finite Toda equation

$$
\tau_{n}^{\prime \prime} \tau_{n}-\left(\tau_{n}^{\prime}\right)^{2}=\tau_{n+1} \tau_{n-1}, \quad n=0, \cdots, N, \quad \tau_{-1}=\tau_{N+1}=0 .
$$

It is easily verified that the boundary condition (4.4) is satisfied by putting

$$
a_{0}^{(0)}=\sum_{i=1}^{N} c_{i} \mathrm{e}^{\mu_{i} t},
$$

where $c_{i}$ and $\mu_{i}(i=1, \ldots, N)$ are arbitrary constants.

It is sometimes convenient to consider the finite Toda equation in the form of (1.3). One reason for this is that the auxiliary linear problem associated with (1.3)

$\alpha_{n-1} \Phi_{n-1}+\beta_{n} \Phi_{n}+\alpha_{n} \Phi_{n+1}=\mu \Phi_{n}, \quad \frac{d \Phi_{n}}{d t}=-\alpha_{n-1} \Phi_{n-1}+\alpha_{n} \Phi_{n+1}$, 
or

$$
\begin{aligned}
& L \Phi=\mu \Phi, \quad \frac{d \Phi}{d t}=B \Phi, \quad \Phi=\left(\begin{array}{c}
\Phi_{1} \\
\Phi_{2} \\
\vdots \\
\Phi_{N}
\end{array}\right) \\
& L=\left(\begin{array}{ccccc}
\beta_{1} & \alpha_{1} & & & \\
\alpha_{1} & \beta_{2} & \alpha_{2} & & \\
& \ddots & \ddots & \ddots & \\
& & \alpha_{N-2} & \beta_{N-1} & \alpha_{N} \\
& & 0 & \alpha_{N-1} & \beta_{N}
\end{array}\right), \quad B=\left(\begin{array}{ccccc}
0 & \alpha_{1} & & & \\
-\alpha_{1} & 0 & \alpha_{2} & & \\
& \ddots & \ddots & \ddots & \\
& & -\alpha_{n-2} & 0 & \alpha_{N} \\
& & -\alpha_{N-1} & 0
\end{array}\right)
\end{aligned}
$$

is self-adjoint[2]. The solutions of the linear problem (2.3) and adjoint linear problem (2.6) are related to $\Phi_{n}$ as

$$
\Psi_{n}=\mathrm{e}^{-\mu t}(-1)^{n} \mathrm{e}^{-\frac{y_{n}}{2}} \Phi_{n}, \quad \Psi_{n}^{*}=\mathrm{e}^{\mu t}(-1)^{n} \mathrm{e}^{\frac{y_{n}}{2}} \Phi_{n}, \quad \mu=-\frac{1}{2} \lambda,(4
$$

respectively.

Remark 4.1 The relationship between entries of determinants and the solutions of linear problems are given by applying Theorem 2.2 as

$$
\Omega_{0}^{(-1)}(t, \lambda)=\left[\frac{\Psi_{1}^{*}(t, \lambda)}{\Psi_{0}^{*}(t, \lambda)}\right]^{(-1)}=\frac{1}{\lambda} \frac{1}{a_{0}^{(0)}} \sum_{i=0}^{\infty} a_{i}^{(0)}(-\lambda)^{-i}
$$

However it is not possible to express (4.11) in terms of the solutions of the linear problem (4.7) $\Phi_{n}$ by using the correspondence (4.10), since $\Phi_{0}$ is not defined for the finite lattice.

\subsection{Results of Moser and Nakamura}

Moser [16] considered $(N, N)$ entry of the resolvent of matrix $L$ :

$$
f(\mu)=(\mu I-L)_{N N}^{-1}=\frac{\Delta_{N-1}}{\Delta_{N}},
$$

where $\Delta_{n}$ is given by

$$
\Delta_{n}=\left|\begin{array}{ccccc}
\mu-\beta_{1} & -\alpha_{1} & & & \\
-\alpha_{1} & \mu-\beta_{2} & -\alpha_{2} & & \\
& \ddots & \ddots & \ddots & \\
& & -\alpha_{n-2} & \mu-\beta_{n-1} & -\alpha_{n-1} \\
& & 0 & -\alpha_{n-1} & \mu-\beta_{n}
\end{array}\right| .
$$

We note that $f(\mu)$ is a rational function in $\mu$, since $\Delta_{n}$ is the $n$-th degree polynomial in $\mu$. By investigating analytic properties of $f(\mu)$, Moser derived the action-angle variables of the finite Toda equation to establish the complete integrability. Nakamura [17] further investigated the expansion of $f(\mu)$ around $\mu=\infty$ to obtain

$$
f(\mu)=\frac{\Delta_{N-1}}{\Delta_{N}}=\frac{1}{\mu} \frac{1}{g_{0}} \sum_{i=0}^{\infty} g_{i}(-2 \mu)^{-i}, \quad g_{i}^{\prime}=g_{i+1},
$$

and claimed that $g_{i}$ are the entries of the determinant formula (4.3), which is quite similar to our result. Let us discuss this result from our point of view. 
By expanding the determinant in (4.13) with respect to $n$-th row, we have the recurrence relation of $\Delta_{n}$

$$
\Delta_{n}=\left(\mu-\beta_{n}\right) \Delta_{n-1}-\alpha_{n-1}^{2} \Delta_{n-2} .
$$

Also, one can show by induction

$$
\Delta_{n}^{\prime}=-2 \alpha_{n}^{2} \Delta_{n-1} \text {. }
$$

Comparing (4.15) and (4.16) with the linear problems (2.3) and (4.7), we have from (1.4) and (4.10)

$$
\Delta_{n}=(-2)^{-n} \Psi_{n+1}=2^{-n} \mathrm{e}^{-\frac{y_{n}}{2}} \Phi_{n+1} .
$$

Now Proposition 2.1 and Theorem 2.2 with $k=N$ yield

$$
\begin{gathered}
\frac{\tau_{N-n}}{\tau_{N}}=\operatorname{det}\left(b_{i+j-2}^{(N)}\right)_{i, j=1, \ldots, n} \\
b_{i}^{(N)}=b_{i-1}^{(N) \prime}+\frac{\tau_{N+1}}{\tau_{N}} \sum_{j=0}^{i-2} b_{j}^{(N)} b_{i-2-j}^{(N)}, \quad b_{0}^{(N)}=\frac{\tau_{N-1}}{\tau_{N}} \\
{\left[\frac{\Psi_{N}(t, \lambda)}{\Psi_{N+1}(t, \lambda)}\right]^{(-1)}=\frac{1}{\lambda} \frac{\tau_{N}}{\tau_{N-1}} \sum_{i=0}^{\infty} b_{i}^{(N)} \lambda^{-i} .}
\end{gathered}
$$

Then taking the boundary condition (4.4) into account, noticing that $\Delta_{n}$ is polynomial of degree $n$ in $\mu=-\frac{\lambda}{2}$, Equation (4.18) can be rewritten by using (4.17) as

$$
\begin{gathered}
\frac{\Delta_{N-1}}{\Delta_{N}}=\frac{1}{\mu} \frac{\tau_{N}}{\tau_{N-1}} \sum_{i=0}^{\infty} b_{i}^{(N)}(-2 \mu)^{-i}, \quad b_{i}^{(N)}=b_{i-1}^{(N) \prime}, \\
\frac{\tau_{N-n}}{\tau_{N}}=\operatorname{det}\left(b_{i+j-2}^{(N)}\right)_{i, j=1, \ldots, n}, \quad b_{0}^{(N)}=\frac{\tau_{N-1}}{\tau_{N}},
\end{gathered}
$$

which is nothing but (4.14). In order to satisfy the boundary condition (4.2) at the left edge of lattice, we choose $b_{0}^{(N)}$ to be sum of $N$ terms of exponential function.

In summary, Nakamura's result may be interpreted as the determinant formula viewed from opposite direction of the lattice. Namely, starting from $n=N$ under normalization $\tau_{N}=1$, it describes such formula that expresses $\tau_{N-n}$ in terms of $n \times n$ determinant. Since $\tau$ function of the finite Toda equation is invariant with respect to inversion of the lattice $(n \rightarrow N-n)$, it is also possible to regard this formula as expressing $\tau_{n}$ as $n \times n$ determinant under the normalization $\tau_{0}=1$. Also, it should be remarked that the resolvent of $L$ appeared because $\Delta_{n}$, principal minor determinant of $\mu I-L$, satisfies the auxiliary linear problem of the finite Toda equation.

Remark 4.2 In order to obtain "normal" determinant formula, we may consider $(1,1)$ entry of the resolvent of $L$

$$
\begin{aligned}
& g(\mu)=(\mu I-L)_{11}^{-1}=\frac{\bar{\Delta}_{1}}{\overline{\bar{\Delta}}_{0}}, \\
& \bar{\Delta}_{n}=\left|\begin{array}{ccccc}
\mu-\beta_{n+1} & -\alpha_{n+1} & & \\
-\alpha_{n+1} & \mu-\beta_{n+2} & -\alpha_{n+2} & & \\
& \ddots & \ddots & \ddots & \\
& & -\alpha_{N-2} & \mu-\beta_{N-1} & -\alpha_{N-1} \\
& & 0 & -\alpha_{N-1} & \mu-\beta_{N}
\end{array}\right|
\end{aligned}
$$


The recurrence relations for $\bar{\Delta}_{n}$ are given by

$$
\bar{\Delta}_{n}=\left(\mu-\beta_{n+1}\right) \bar{\Delta}_{n+1}-\alpha_{n+2}^{2} \bar{\Delta}_{n+2}, \quad \bar{\Delta}_{n}^{\prime}=2 \alpha_{n}^{2} \bar{\Delta}_{n+1},
$$

which implies

$$
\bar{\Delta}_{n}=(-2)^{n} \Psi_{n}^{*}=2^{n} \mathrm{e}^{\frac{y_{n}}{2}} \Phi_{n} .
$$

Therefore Proposition 2.1 and Theorem 2.2 yield

$$
\begin{gathered}
\frac{\bar{\Delta}_{1}}{\bar{\Delta}_{0}}=\frac{1}{\mu} \frac{\tau_{0}}{\tau_{1}} \sum_{i=0}^{\infty} a_{i}^{(0)}(2 \mu)^{-i}, \quad a_{i}^{(0)}=a_{i-1}^{(0) \prime}, \quad a_{0}^{(0)}=\frac{\tau_{1}}{\tau_{0}}, \\
\frac{\tau_{n}}{\tau_{0}}=\operatorname{det}\left(a_{i+j-2}^{(0)}\right)_{i, j=1, \ldots, n} .
\end{gathered}
$$

\section{Concluding remarks}

In this article we have established the relationship between the Hankel determinant formula and the auxiliary linear problem. We have also presented a compact formula of the $\tau$ function in the framework of the KP theory. The similar phenomena that have been observed in the Painlevé II and IV equation can be recovered from this result. We have also pointed out that Moser and Nakamura's result on the finite Toda equation can be understood naturally in our framework.

Since the Toda equation can be seen in various context, we expect that the structure presented in this article can be observed in wide area of physical and mathematical sciences. Moreover, it might be an intriguing problem to study whether similar phenomenon can be observed or not for the periodic lattice, where the theta functions play the role of the $\tau$ functions.

\section{Acknowledgments}

The authors would like to express their sincere thanks to N. Joshi for fruitful discussions. They also thank Y. Nakamura and G. Carlet for helpful conversation. This research was partially supported by MIMS, the Manchester Institute for Mathematical Sciences. KK is supported by the JSPS Grant-in-Aid for Scientific Research (B)15340057 and (A)16204007. He also acknowledges the support by the 21st Century COE program at the Faculty of Mathematics, Kyushu University.

\section{References}

[1] Fadeev L D, Takhtajan L A and Tarasov V O 1983 Local Hamiltonians for integrable quantum models on a lattice Teoret. Mat. Fiz. 57 163-81

[2] Flaschka H 1974 The Toda lattice. I. Existence of integrals Phys. Rev. B 9 1924-5

[3] Goto H and Kajiwara K 2005 Generating function related to the Okamoto polynomials for the Painlevé IV equation Bull. Aust. Math. Soc. 71 517-26

[4] Hirota R, Ito M and Kako F 1988 Two-dimensional Toda lattice equations Progr. Theoret. Phys. Suppl. $9442-58$

[5] Hirota R 1988 Toda molecule equations Algebraic analysis Vol. I (Boston: Academic Press) p 203-16

[6] Hirota R 1987 Discrete Two-dimensional Toda molecule equation J. Phys. Soc. Jpn. 56 4285-8

[7] Iwasaki K, Kajiwara K and Nakamura T 2002 Generating function associated with the rational solutions of the Painlevé II equation J. Phys. A: Math. Gen. 35 L207-11

[8] Jimbo M and Miwa T 1981 Monodoromy preserving deformation of linear ordinary differential equations with rational coefficients.II Physica 2D 407-48 
[9] Jimbo M and Miwa T 1983 Solitons and infinite dimensional Lie algebras Publ. RIMS $19943-$ 1001

[10] Joshi N, Kajiwara K and Mazzocco M 2004 Generating function associated with the determinant formula for solutions of the Painlevé II equation Astérisque 274 67-78

[11] Joshi N, Kajiwara K and Mazzocco M 2006 Generating function associated with the determinant Formula for solutions of the Painlevé IV Equation to appear in Funkcial. Ekvac.

[12] Kajiwara K, Masuda T, Noumi M, Ohta Y and Yamada Y 2001 Determinant formulas for the Toda and discrete Toda equations Funkcial. Ekvac. 44 291-307

[13] Leznov A N and Saveliev M V 1981 Theory of group representations and integration of nonlinear systems $x_{a, z \bar{z}}=\exp (k x)_{a}$ Physica $3 D$ 62-72

[14] Miwa T 1982 On Hirota's difference equations Proc. J. Acad. 58 Ser. A 9-12

[15] Miwa T, Jimbo M and Date E 2000 SOLITONS, Differential equations, symmetries and infinite dimensional algebras (Cambridge tracts in mathematics Vol. 135) (Cambridge: Cambridge University Press)

[16] Moser J 1975 Finitely many mass points on the line under the influence of an exponential potential - an integrable system, Dynamical Systems, Theory and Applications (Lecture Notes in Physics Vol 38) ed J Moser (Berlin: Springer-Verlag) p 467-97

[17] Nakamura Y 1994 A tau-function of the finite nonperiodic Toda lattice, Phys. Lett. A195 346-50

[18] Noumi M 2004 Painlevé equations through symmetry (Translations of mathematical monographs Vol 223) (Providence: American Mathematical Society)

[19] Ohta Y, Satsuma J, Takahashi D and Tokihiro T 1988 An elementary introduction to Sato theory Progr. Theoret. Phys. 80 210-41

[20] Okamoto K 1987 Studies on the Painlevé equations. I. Sixth Painlevé equation PI. Ann. Mat. Pura Appl. 146 337-81

[21] Okamoto K 1987 Studies on the Painlevé equations. II. Fifth Painlevé equation PV, Japan. J. Math. 13 47-76

[22] Okamoto K 1986 Studies on the Painlevé equations. III. Second and fourth Painlevé equations, $P_{\mathrm{II}}$ and $P_{\mathrm{IV}}$, Math. Ann. $275221-55$

[23] Okamoto K 1987 Studies on the Painlevé equations. IV. Third Painlevé equation $P_{\text {III }}$ Funkcial. Ekvac. 30 305-32

[24] Toda M 1967 Vibration of a Chain with Nonlinear Interaction J. Phys. Soc. Jpn. 22 431-6

[25] Ueno K and Takasaki K 1984 Toda lattice hierarchy Group representations and systems of differential equations (Adv. Stud. Pure Math. Vol.4)(Amsterdam: North-Holland) p 1-95 


\section{List of MHF Preprint Series, Kyushu University 21st Century COE Program \\ Development of Dynamic Mathematics with High Functionality}

\section{MHF2005-1 Hideki KOSAKI}

Matrix trace inequalities related to uncertainty principle

MHF2005-2 Masahisa TABATA

Discrepancy between theory and real computation on the stability of some finite element schemes

MHF2005-3 Yuko ARAKI \& Sadanori KONISHI

Functional regression modeling via regularized basis expansions and model selection

MHF2005-4 Yuko ARAKI \& Sadanori KONISHI

Functional discriminant analysis via regularized basis expansions

MHF2005-5 Kenji KAJIWARA, Tetsu MASUDA, Masatoshi NOUMI, Yasuhiro OHTA \& Yasuhiko YAMADA

Point configurations, Cremona transformations and the elliptic difference Painlevé equations

MHF2005-6 Kenji KAJIWARA, Tetsu MASUDA, Masatoshi NOUMI, Yasuhiro OHTA \& Yasuhiko YAMADA

Construction of hypergeometric solutions to the $q$ - Painlevé equations

MHF2005-7 Hiroki MASUDA

Simple estimators for non-linear Markovian trend from sampled data:

I. ergodic cases

MHF2005-8 Hiroki MASUDA \& Nakahiro YOSHIDA

Edgeworth expansion for a class of Ornstein-Uhlenbeck-based models

MHF2005-9 Masayuki UCHIDA

Approximate martingale estimating functions under small perturbations of dynamical systems

MHF2005-10 Ryo MATSUZAKI \& Masayuki UCHIDA

One-step estimators for diffusion processes with small dispersion parameters from discrete observations

MHF2005-11 Junichi MATSUKUBO, Ryo MATSUZAKI \& Masayuki UCHIDA

Estimation for a discretely observed small diffusion process with a linear drift

MHF2005-12 Masayuki UCHIDA \& Nakahiro YOSHIDA

AIC for ergodic diffusion processes from discrete observations 
MHF2005-13 Hiromichi GOTO \& Kenji KAJIWARA

Generating function related to the Okamoto polynomials for the Painlevé IV equation

MHF2005-14 Masato KIMURA \& Shin-ichi NAGATA

Precise asymptotic behaviour of the first eigenvalue of Sturm-Liouville problems with large drift

MHF2005-15 Daisuke TAGAMI \& Masahisa TABATA

Numerical computations of a melting glass convection in the furnace

MHF2005-16 Raimundas VIDŪNAS

Normalized Leonard pairs and Askey-Wilson relations

MHF2005-17 Raimundas VIDŪNAS

Askey-Wilson relations and Leonard pairs

MHF2005-18 Kenji KAJIWARA \& Atsushi MUKAIHIRA

Soliton solutions for the non-autonomous discrete-time Toda lattice equation

MHF2005-19 Yuu HARIYA

Construction of Gibbs measures for 1-dimensional continuum fields

MHF2005-20 Yuu HARIYA

Integration by parts formulae for the Wiener measure restricted to subsets in $\mathbb{R}^{d}$

MHF2005-21 Yuu HARIYA

A time-change approach to Kotani's extension of Yor's formula

MHF2005-22 Tadahisa FUNAKI, Yuu HARIYA \& Mark YOR

Wiener integrals for centered powers of Bessel processes, I

MHF2005-23 Masahisa TABATA \& Satoshi KAIZU

Finite element schemes for two-fluids flow problems

MHF2005-24 Ken-ichi MARUNO \& Yasuhiro OHTA

Determinant form of dark soliton solutions of the discrete nonlinear Schrödinger equation

MHF2005-25 Alexander V. KITAEV \& Raimundas VIDŪNAS

Quadratic transformations of the sixth Painlevé equation

MHF2005-26 Toru FUJII \& Sadanori KONISHI

Nonlinear regression modeling via regularized wavelets and smoothing parameter selection

MHF2005-27 Shuichi INOKUCHI, Kazumasa HONDA, Hyen Yeal LEE, Tatsuro SATO, Yoshihiro MIZOGUCHI \& Yasuo KAWAHARA

On reversible cellular automata with finite cell array 
MHF2005-28 Toru KOMATSU

Cyclic cubic field with explicit Artin symbols

MHF2005-29 Mitsuhiro T. NAKAO, Kouji HASHIMOTO \& Kaori NAGATOU

A computational approach to constructive a priori and a posteriori error estimates for finite element approximations of bi-harmonic problems

MHF2005-30 Kaori NAGATOU, Kouji HASHIMOTO \& Mitsuhiro T. NAKAO Numerical verification of stationary solutions for Navier-Stokes problems

MHF2005-31 Hidefumi KAWASAKI

A duality theorem for a three-phase partition problem

MHF2005-32 Hidefumi KAWASAKI

A duality theorem based on triangles separating three convex sets

MHF2005-33 Takeaki FUCHIKAMI \& Hidefumi KAWASAKI

An explicit formula of the Shapley value for a cooperative game induced from the conjugate point

MHF2005-34 Hideki MURAKAWA

A regularization of a reaction-diffusion system approximation to the two-phase Stefan problem

MHF2006-1 Masahisa TABATA

Numerical simulation of Rayleigh-Taylor problems by an energy-stable finite element scheme

MHF2006-2 Ken-ichi MARUNO \& G R W QUISPEL

Construction of integrals of higher-order mappings

MHF2006-3 Setsuo TANIGUCHI

On the Jacobi field approach to stochastic oscillatory integrals with quadratic phase function

MHF2006-4 Kouji HASHIMOTO, Kaori NAGATOU \& Mitsuhiro T. NAKAO

A computational approach to constructive a priori error estimate for finite element approximations of bi-harmonic problems in nonconvex polygonal domains

MHF2006-5 Hidefumi KAWASAKI

A duality theory based on triangular cylinders separating three convex sets in $R^{n}$

MHF2006-6 Raimundas VIDŪNAS

Uniform convergence of hypergeometric series

MHF2006-7 Yuji KODAMA \& Ken-ichi MARUNO

N-Soliton solutions to the DKP equation and Weyl group actions 
MHF2006-8 Toru KOMATSU

Potentially generic polynomial

MHF2006-9 Toru KOMATSU

Generic sextic polynomial related to the subfield problem of a cubic polynomial

MHF2006-10 Shu TEZUKA \& Anargyros PAPAGEORGIOU

Exact cubature for a class of functions of maximum effective dimension

MHF2006-11 Shu TEZUKA

On high-discrepancy sequences

MHF2006-12 Raimundas VIDŪNAS

Detecting persistent regimes in the North Atlantic Oscillation time series

MHF2006-13 Toru KOMATSU

Tamely Eisenstein field with prime power discriminant

MHF2006-14 Nalini JOSHI, Kenji KAJIWARA \& Marta MAZZOCCO

Generating function associated with the Hankel determinant formula for the solutions of the Painlevé IV equation

MHF2006-15 Raimundas VIDŪNAS

Darboux evaluations of algebraic Gauss hypergeometric functions

MHF2006-16 Masato KIMURA \& Isao WAKANO

New mathematical approach to the energy release rate in crack extension

MHF2006-17 Toru KOMATSU

Arithmetic of the splitting field of Alexander polynomial

MHF2006-18 Hiroki MASUDA

Likelihood estimation of stable Lévy processes from discrete data

MHF2006-19 Hiroshi KAWABI \& Michael RÖCKNER

Essential self-adjointness of Dirichlet operators on a path space with Gibbs measures via an SPDE approach

MHF2006-20 Masahisa TABATA

Energy stable finite element schemes and their applications to two-fluid flow problems

MHF2006-21 Yuzuru INAHAMA \& Hiroshi KAWABI

Asymptotic expansions for the Laplace approximations for Itô functionals of Brownian rough paths

MHF2006-22 Yoshiyuki KAGEI

Resolvent estimates for the linearized compressible Navier-Stokes equation in an infinite layer 
MHF2006-23 Yoshiyuki KAGEI

Asymptotic behavior of the semigroup associated with the linearized

compressible Navier-Stokes equation in an infinite layer

MHF2006-24 Akihiro MIKODA, Shuichi INOKUCHI, Yoshihiro MIZOGUCHI \& Mitsuhiko FUJIO

The number of orbits of box-ball systems

MHF2006-25 Toru FUJII \& Sadanori KONISHI

Multi-class logistic discrimination via wavelet-based functionalization and model selection criteria

MHF2006-26 Taro HAMAMOTO, Kenji KAJIWARA \& Nicholas S. WITTE

Hypergeometric solutions to the $q$-Painlevé equation of type $\left(A_{1}+A_{1}^{\prime}\right)^{(1)}$

MHF2006-27 Hiroshi KAWABI \& Tomohiro MIYOKAWA

The Littlewood-Paley-Stein inequality for diffusion processes on general metric spaces

MHF2006-28 Hiroki MASUDA

Notes on estimating inverse-Gaussian and gamma subordinators under highfrequency sampling

MHF2006-29 Setsuo TANIGUCHI

The heat semigroup and kernel associated with certain non-commutative harmonic oscillators

MHF2006-30 Setsuo TANIGUCHI

Stochastic analysis and the KdV equation

MHF2006-31 Masato KIMURA, Hideki KOMURA, Masayasu MIMURA, Hidenori MIYOSHI, Takeshi TAKAISHI \& Daishin UEYAMA

Quantitative study of adaptive mesh FEM with localization index of pattern

MHF2007-1 Taro HAMAMOTO \& Kenji KAJIWARA

Hypergeometric solutions to the $q$-Painlevé equation of type $A_{4}^{(1)}$

MHF2007-2 Kouji HASHIMOTO, Kenta KOBAYASHI \& Mitsuhiro T. NAKAO

Verified numerical computation of solutions for the stationary Navier-Stokes equation in nonconvex polygonal domains

MHF2007-3 Kenji KAJIWARA, Marta MAZZOCCO \& Yasuhiro OHTA

A remark on the Hankel determinant formula for solutions of the Toda equation 